\title{
Fragile X-associated neuropsychiatric disorders: a case report
}

\author{
Maria Melinda G Tan 1,2,3,4, Jeanne Barbara S Dy 1,2,5,4, Maria Jimena Salcedo-Arellano ${ }^{1,6}$, \\ Flora Tassone $^{1,7}$ \& Randi J Hagerman*,1,6 \\ ${ }^{1}$ Medical Investigation of Neurodevelopmental Disorders (MIND) Institute, University of California Davis Health, Sacramento, CA \\ 95817, USA \\ ${ }^{2}$ MedMom Institute for Human Development, Pasig City, NCR, Philippines \\ ${ }^{3}$ Department of Psychology, University of the Philippines Diliman, Quezon City, NCR, Philippines \\ ${ }^{4}$ Ateneo de Manila University School of Medicine \& Public Health, Pasig City, NCR, Philippines \\ ${ }^{5}$ Department of Pediatrics, The Medical City, Ortigas Avenue, Pasig City, NCR, Philippines \\ ${ }^{6}$ Department of Pediatrics, University of California Davis School of Medicine, Sacramento, CA 95817, USA \\ ${ }^{7}$ Department of Biochemistry \& Molecular Medicine, University of California Davis Medical Center, Sacramento, CA 95817, USA \\ *Author for correspondence: rjhagerman@ucdavis.edu
}

Mutations in the FMR1 gene have been associated with developmental or neurodegenerative disorders. The full mutation ( $>200$ CGG repeats) results in Fragile $X$ syndrome, the most common inherited cause of intellectual disability, while the premutation (55-200 CGG repeats) can lead to a range of problems including fragile X-associated tremor/ataxia syndrome (FXTAS). Recently, a new distinctive name was proposed to recognize the associated disorders commonly found in premutation carriers and extensively reported in co-morbidities studies: fragile X-associated neuropsychiatric disorders (FXAND). This paper will present a case report of a female premutation carrier with a complex psychiatric history, chronic pain, and sleep disturbances consistent with Fragile X-associated neuropsychiatric disorders.

First draft submitted: 3 December 2018; Accepted for publication: 14 March 2019; Published online: 24 May 2019

Keywords: ADHD • anxiety $\bullet$ chronic pain $\bullet$ depression $\bullet$ FMR1 • Fragile X-associated Neuropsychiatric Disorders $\bullet$ FXAND • premutation carrier $\bullet$ psychiatric $\bullet$ sleep disorders

Fragile X syndrome is caused by the amplification of CGG repeats in the fragile X mental retardation 1 (FMRI) gene. Mutations in the FMRI gene are associated with developmental disorders in children and neurodegenerative problems in older populations. The full mutation (>200 CGG repeats) in the FMR1 gene results in the fragile $\mathrm{X}$ syndrome (FXS), which is the most common inherited cause of intellectual disability and autism spectrum disorder. The premutation (55-200 CGG repeats), which is found in approximately 1 in 200 women and 1 in 400 men [1], can also result in problems linked to the elevated levels of the FMR1 mRNA leading to mRNA toxicity and occasionally mildly deficient FMRP levels [2]. Associated disorders among premutation carriers have been previously named, including fragile X-associated primary ovarian insufficiency (FXPOI), a condition that occurs in an estimated $16-20 \%$ of carriers and characterized by menopause before 40 years old [3,4], as well as fragile $\mathrm{X}$-associated tremor/ataxia syndrome (FXTAS), a neurodegenerative condition which is diagnosed in about $16 \%$ of females and $40 \%$ of older male premutation carriers [5-7]. However, there have been increasing reports of female premutation carriers who present with a variety of neurologic, psychiatric, sleep and autoimmune conditions, which has recently been called fragile X-associated neuropsychiatric disorders (FXAND). This group of conditions, primarily neuropsychiatric in presentation, affects approximately $50 \%$ of carriers [8]. This case report illustrates many of the conditions associated with FXAND in a woman with the premutation.

\section{Materials \& methods}

This patient was evaluated as part of the Fragile X Treatment and Research Center located at the University of California Davis MIND Institute for management of Fragile X disorders. Detailed medical history, physical and neurological examinations, and genetic testing were done. The neurological examination was carried out by

Future $\because$ Medicine 
a medical doctor and included evaluating cranial nerve function, reflexes, muscle tone, rigidity, sensation, gait, coordination and the presence of abnormal movements. The patient signed an informed consent form approved by the Institutional Review Board (IRB) to have her case history published. Here, we describe the patient's medical history, neuropsychiatric symptoms, genetic information, and findings on medical and neurological evaluations.

\section{Molecular measures}

CGG repeat sizes and activation ratio were determined using PCR amplification and southern blot analysis gene according to the conditions described in Tassone et al. (2008) and Filipovic-Sadic (2010) [9,10]. FMR1 mRNA expression levels were determined by quantitative reverse transcription polymerase chain reaction (RT-PCR) as detailed in Tassone et al. (2000) [2].

\section{Case report}

This is the case of a 55-year-old woman, diagnosed as a premutation carrier with two alleles of 29 and 70 CGG repeats, an activation ratio of 0.62 (which expresses the percent of cell carrying the normal allele on the active X chromosome), and an FMR1 mRNA expression level of 1.9 (0.04)-fold higher compared with controls. She presented with longstanding problems in anxiety, depression, inattention, chronic pain and opioid use. Maternal and birth histories were unremarkable while developmental milestones were achieved within normal limits. Retrospectively, she reported experiencing anxiety and 'panic attacks' in early childhood, as far back as 3 years old, and these persisted into adulthood. She initially performed well in school despite her dislike of reading, but experienced more problems in attention, organization, and academics in high school. These resulted in poor grades and increased parent-child conflict although no diagnosis or treatment were given at that time. These events, alongside living with a high-achieving sibling who was not a premutation carrier, exacerbated her anxiety and reportedly led to a negative self image.

She drank alcohol as an adolescent to ostensibly 'self medicate' her anxiety and stress due to her poor self image and conflict with parents regarding her academic difficulties. She was eventually diagnosed with depression and anxiety in her 20s, and prescribed fluoxetine with beneficial effects. Bupropion $\mathrm{Hcl}$ was also given for her depression between 2000 to 2017 but it eventually stopped working at a dose of $300 \mathrm{mg} /$ day. Throughout adulthood, she continued to take fluoxetine $40 \mathrm{mg} /$ day.

She experimented with street amphetamines to address her symptoms of inattention. It was only recently that she was diagnosed with attention-deficit/hyperactivity disorder (ADHD) and prescribed Adderall $20 \mathrm{mg} / \mathrm{day}$, a preparation of mixed amphetamine salts which improves attention.

Opioid abuse has also been an intermittent problem for many years. She was first treated with hydrocodone after experiencing postoperative pain from a caesarean section delivery, alleviating both her pain and depression. Hydrocodone use subsequently increased after stressful events successively occurred including the death of her father, her son's diagnosis of autism spectrum disorder (ASD), and her premutation carrier diagnosis. She sought treatment for alcohol and opioid use in an outpatient program with temporary improvements, but later relapsed, resulting in her admission to an inpatient rehabilitation program. She experienced another relapse after a year when her mother passed away. Currently, she reportedly limits her alcohol intake to two drinks a day. She still uses hydrocodone $5 \mathrm{mg}$ three times a day since her depression reportedly becomes so severe when she stops taking hydrocodone.

She experienced intermittent low back pain for a period of approximately 20 years, which she attributed to her involvement in gymnastics when she was younger. But in the last 2 years, she has had chronic pain problems described as constant pain in her neck and shoulders, right arm and trapezius muscles. There is no history of back surgery and she was never diagnosed with fibromyalgia. A rheumatologist consultation was done several years ago and a blood test revealed a positive antinuclear antibodies (ANA) titer at 1:360.

She reportedly had 'tics' in childhood described as chewing and repetitively tapping on a table. These 'tics' decreased after puberty but reappeared with her stress about her mother's passing. Her tics worsened after Adderall dosage was increased to $30 \mathrm{mg}$, thus, the dose was kept at $20 \mathrm{mg}$.

She had one child who was also a premutation carrier (133 CGG repeats). She never had symptoms of premature ovarian insufficiency and the onset of menopause was at age 52. Movement-related concerns were reported at age 47 characterized by intermittent tremors while manipulating a computer mouse, although tremors have recently improved in that she does not notice these. There are no reported symptoms of ataxia although she has tripped a 
Table 1. Summary of medical history.

\begin{tabular}{|c|c|c|}
\hline Diagnosis & Age of onset & Medication/dose per day \\
\hline FMR1 premutation (70 CGG repeats) & $\mathrm{N} / \mathrm{A}$ & \\
\hline Anxiety & Childhood & Fluoxetine $40 \mathrm{mg}$ (ongoing) \\
\hline Social phobia & Childhood & \\
\hline Specific phobia (elevators) & Childhood & \\
\hline Depression & $20 \mathrm{~s}$ & $\begin{array}{l}\text { Fluoxetine } 40 \text { mg (ongoing) } \\
\text { Bupropion } 300 \text { mg (2010-2017) }\end{array}$ \\
\hline Substance abuse & $30 \mathrm{~s}$ & $\begin{array}{l}\text { Alcohol 2+ drinks (ongoing) } \\
\text { Hydrocodone } 15 \text { mg (ongoing) }\end{array}$ \\
\hline Chronic pain & $30 \mathrm{~s}$ & \\
\hline ADHD & $40 \mathrm{~s}$ & Adderall 20 mg (ongoing) \\
\hline Sleep disorder & $50 \mathrm{~s}$ & Trazodone $50-75 \mathrm{mg}$ (ongoing) \\
\hline Memory deficit & $50 \mathrm{~s}$ & \\
\hline
\end{tabular}

few times without falling down. She describes numbness in her fingertips but not in her feet. She also experiences chronic fatigue, which is alleviated by Adderall.

She has had vasovagal episodes during pregnancy and more recently experienced orthostatic hypotension after standing up quickly from a sitting position.

Cognitive issues were also reported, characterized by memory problems and word retrieval difficulties, which began originally at age 38 but worsened in her 50 s.

She reported experiencing 'manic behaviors' in the past, consistent with the criteria for bipolar disorder in partial remission on the Structured Clinical Interview for the DSM-5 (SCID-5). She had sleep problems due to racing thoughts although her sleep improved with intake of trazodone 50-75 mg at night. She refused benzodiazepines for her sleep problems because of her concern that these could be addictive. She also reported frequent snoring but has not had a polysomnogram to diagnose sleep apnea.

Her current SCID-5 evaluation documents subthreshold depression on fluoxetine, past polydrug abuse, and fulfills the DSM-5 criteria for social phobia and specific phobia for elevators.

She continues on fluoxetine $40 \mathrm{mg} /$ day, Adderall $20 \mathrm{mg} /$ day, trazodone $75 \mathrm{mg}$ at bedtime and hydrocodone $5 \mathrm{mg}$ three times a day. She has an exercise regimen of at least 4 hours per week and works as a personal trainer, recognizing the importance of exercise for physical and mental health.

On neurological exam, there were no obvious tremors with finger to nose touching. With positioning, there was a slight, subclinical tremor in the right hand more than the left. There was no resting tremor. Vibration sense was normal in all extremities. Primitive reflexes were absent. On tandem gait, she had to leave some space in between both feet to maintain her balance while executing the motor task. She had an MRI that did not demonstrate any signs of atrophy or the middle cerebellar peduncle sign, but it showed slight white matter disease in the pons on the right hemisphere and in the insula. Her corpus callosum was without atrophy but with slight involvement of the splenium and hyperintensities on T2, representing white matter disease.

She does not meet diagnostic criteria for FXTAS; however, most of her symptoms involve neuropsychiatric problems, therefore putting her in the FXAND diagnostic category. See Table 1 for a summary of her medical history.

\section{Discussion}

Here, we present a case of an adult female premutation carrier with a lifelong history of anxiety and intermittent depression consistent with FXAND [8] who does not meet the criteria for FXTAS [11,12]. Her case will add to the clinical data on FXAND, a recently described fragile X-associated disorder.

FXAND is a proposed umbrella term that represents the neuropsychiatric conditions associated with fragile $\mathrm{X}$ premutation carriers. It is not limited to one entity but includes various neuropsychiatric disorders. It primarily presents as neuropsychiatric symptoms in premutation carriers who do not have the clinical signs of FXTAS, such as significant action tremor and/or cerebellar ataxia, in addition to white matter disease on MRI and white matter hyperintensities in the middle cerebellar peduncles (MCP) [8,11,12]. Neuropsychiatric problems in FXAND emerge before the neurological problems develop in FXTAS and onset is typically at an earlier age than FXTAS [8]. 
Due to the presence of a 70 CGG repeat allele, she was subsequently diagnosed as a premutation carrier. The relationship between CGG repeats and the prevalence of major depressive disorder is curvilinear. This means that the middle range of $70-100$ repeats confers the greatest risk, while repeats on the lower end and higher end of the premutation range confer lower risks of psychiatric problems [13].

As a premutation carrier with major depression that meets the DSM-5 criteria, she is labeled as suffering from FXAND due to the association of her depression with the premutation carrier status. Moreover, she experienced a constellation of neuropsychiatric problems that is greater in number and severity than the usual patient with FXAND. The number of neuropsychiatric conditions present in this patient further strengthens the association of these disorders with the umbrella term FXAND seen in a proportion of premutation carriers.

The neuropathological mechanisms that lead to neuropsychiatric disorders of FXAND are still unclear, although the mechanisms underlying FXTAS may shed some light into this. Problems associated with the premutation are related to the RNA toxicity because of elevated FMR1-mRNA. Neurons of premutation carriers are more vulnerable to environmental toxins [12] and more neuronal death is seen in cell cultures [14]. Ca ++ dysregulation and elevated cytoplasmic $\mathrm{Ca}++$ levels are also present in premutation neurons [15], which may be associated with the mitochondrial dysfunction that worsens at the onset of FXTAS [16,17]. Chronic DNA damage repair and the formation of FMRpolyG, a toxic protein [18], are also related to the toxicity of the premutation that leads to the neurodegenerative process in FXTAS. Similar neuropathological mechanisms may be found in FXAND resulting in the dysfunction of neural systems responsible for behavior and emotion regulation. A recent paper by Brown et al. (2019) compared 17 male carriers without FXTAS to 17 age-matched controls (ages 24-70) and found higher rates of psychopathology in the carriers than controls. In addition, functional magnetic resonance imaging (fMRI) studies demonstrated remarkably lowered activation patterns to emotional stimuli compared with controls. These findings did not correlate or change with age. Rather, they appear to represent life span problems of a neurodevelopmental origin perhaps related to neuronal connectivity deficits in carriers [19].

The patient reported experiencing anxiety from childhood and intermittent depression with multiple psychosocial stressors, consistent with findings about the developmental trajectory and risk factors associated with the premutation. Higher rates of anxiety disorder are seen among $F M R 1$ premutation carriers compared to the general population. A lifetime prevalence for anxiety disorder in males with FXTAS is 50\% [20], and about $40-47 \%$ in carriers without FXTAS [21]. These anxiety symptoms are typically present in adolescence, and approximately $50 \%$ of female premutation carriers would have met criteria for anxiety disorder before 18 years old [22]. Another study looking at male and female carriers between 4 and 22 years old estimated that $70 \%$ fulfilled the criteria for at least one type of anxiety disorder in contrast to $9.8 \%$ in the general population. Psychosocial stressors heighten the risk for anxiety, including knowing that one is a carrier [23], or parenting a child with problem behaviors [22]. In premutation carriers with FXTAS, anxiety is associated with progressive decline in cognitive abilities and hippocampal atrophy, as neurodegenerative processes detrimentally impact on neural systems underpinning executive control and emotion regulation [20]. Depression is also frequently reported by carriers. The lifetime prevalence of major depressive disorder is approximately $40 \%$ among premutation carriers without FXTAS [22,24] and with FXTAS [20]. Male premutation carriers without FXTAS are more likely to rate themselves higher on depression scales relative to the normative population [20], with depressive symptoms predicted to worsen over time [25]. Depressive symptoms are also aggravated by chronic stressors including parenting a child with FXS [22] and comorbid medical conditions experienced by the carrier.

The patient had ADHD symptoms since childhood, but was not diagnosed nor treated then, so she turned to street amphetamines to self medicate. ADHD is common in premutation carriers [25] and occurs in over 50\% for those who present clinically. Attention deficits are also more frequently detected in carriers than their noncarrier sibling - with more males (41\%) demonstrating problems compared to females (18.5\%) [26]. Hyperactivity may decline over time although inattentive symptoms may endure [27]. The FMR1 mutation is a factor that may increase the risk for ADHD symptoms so this is included in FXAND [28].

Pain symptoms are often reported by adult premutation carriers. Fibromyalgia can occur in up to $40 \%$ of women with FXTAS [29]. The mechanism through which female premutation carriers develop fibromyalgia is via the alteration of pain neurotransmission through pain dysregulation, resulting from the damaging effects of increased rates of transcription of expanded FMR1 mRNA [30]. Both the ADHD and pain symptoms have led to chronic substance abuse in this history and these problems have been mentioned in the literature regarding co-morbidities in carriers [31,32]. They have also been included in diagnostic entities covered by FXAND. Additionally, higher 
rates of alcohol abuse have been documented in carriers, placing them at further risk for other neuropsychiatric co-morbidities [33].

The patient reported reading difficulties due to inattention and experienced academic problems, although she was never evaluated or given a diagnosis of a learning disorder. While not necessarily psychiatric, her learning problem was a major stressor during adolescence which could have increased her risk for anxiety and depression. She also reported cognitive issues as an adult related to memory and word retrieval problems. Cognitive profiles of premutation carriers are varied, in that some may have normal cognitive functioning while others may present with a lower verbal intelligence quotient (IQ) scores. Information about learning disorders is minimal although arithmetic difficulties have been reported in females premutation carriers [34].

Chronic fatigue is common among carriers even before the onset of FXTAS, probably secondary to the mitochondrial dysfunction $[35,36$. Moreover, CNS volumetric changes occur throughout the lifetime in carriers particularly in the cerebellum and brainstem. Some of the patient's symptoms such as intermittent tremor are likely related to the neurological findings on MRI. White matter disease in the CNS can occur even before the symptoms of FXTAS emerge [11], and this patient demonstrates white matter disease in the insula and in the splenium of the corpus callosum.

Sleep disturbances in premutation carriers are also frequent. These may be caused by their anxiety and depression, or these could also be associated with the GABA deficits documented in premutation carriers [37] secondary to a mild FMRP deficit or to the RNA toxicity. There is also an increased risk of sleep apnea in premutation carriers [38], which affects restorative sleep. This may play a role in the chronic fatigue they experience and predispose them to more attention and memory problems.

In regard to the patient's son, although he does not have the full mutation, he has ASD which is seen in boys with the premutation [25]. This also comes under the diagnoses included in FXAND [8].

In sum, this is a case of a premutation carrier with complex neuropsychiatric symptoms consistent with FXAND that missed early detection leading to delayed or inconsistent interventions. Her neuropsychiatric co-morbidities, together with the challenges of parenting, the impact of her premutation diagnosis, and her experience of losing her parents, resulted in complex stresses that exacerbated the neurobiological vulnerabilities associated with the premutation.

\section{Conclusion}

This case report provides a clinical example of complex neuropsychiatric symptoms associated with FXAND, which can be severe, lifelong, and require intensive counseling and pharmacotherapy. Individuals with the FMRI premutation are at an increased risk for developing anxiety, depression, ADHD, substance abuse, and medical issues related to pain, chronic fatigue, sleep, and autoimmune problems. However, the association of these psychiatric problems with the premutation is not always acknowledged, resulting in missed diagnoses or suboptimal treatment. In this case, the patient consequently experienced longstanding neuropsychiatric issues complicated by substance abuse. This underscores the need for early identification and intervention for carriers at risk.

The diagnosis of FXAND requires FMR1 DNA testing to identify if one is a premutation carrier (55-200 CGG repeats), and a psychiatric evaluation to determine the presence of a neuropsychiatric disorder. These, along with genetic counseling, can guide treatment. Timely diagnosis is crucial due to the premutation carrier's likelihood of developing the co-morbidities discussed here, as well as having children with an FMR1 mutation and accompanying neuropsychiatric or neurodevelopmental disorders. Conversely, individuals presenting with mood or anxiety disorders and a family history of FXS, developmental delay, intellectual disability or autism spectrum disorder should also be screened [33,39].

Further investigations into the neurobiological and molecular underpinnings of FXAND are necessary to clarify the mechanisms leading to these neuropsychiatric conditions and to fine tune treatments that alleviate these symptoms. 
authors have no other relevant affiliations or financial involvement with any organization or entity with a financial interest in or financial conflict with the subject matter or materials discussed in the manuscript. This includes employment, consultancies, honoraria, stock ownership or options, expert testimony, grants or patents received or pending, or royalties.

No writing assistance was utilized in the production of this manuscript.

\section{Ethical disclosure}

The authors state that they have obtained an IRB-approved informed consent from the participant involved.

\section{Authors' contributions}

All of the authors participated in drafting the manuscript. RJ Hagerman additionally revised it critically for important intellectual content. All of the authors gave final approval of the version to be submitted.

\section{Open access}

This work is licensed under the Creative Commons Attribution-NonCommercial-NoDerivatives 4.0 Unported License. To view a copy of this license, visit: http://creativecommons.org/licenses/by-nc-nd/4.0/

\section{Executive summary}

- Fragile X-associated neuropsychiatric disorder (FXAND) is an umbrella term that represents the neuropsychiatric disorders associated with the FMR1 premutation, which include anxiety, depression, ADHD, obsessive compulsive disorder, chronic fatigue, insomnia, chronic pain and/or fibromyalgia.

- The neuropathological mechanisms leading to neuropsychiatric disorders of FXAND may be related to mitochondrial dysfunction secondary to RNA toxicity caused by elevated FMR1-mRNA. These are likely developmental and not neurodegenerative.

- FXAND can be mild or severe, and lifelong. Problems associated with FXAND usually respond well to counseling and pharmacotherapy.

- Anxiety, depression, and attention-deficit/hyperactivity disorder (ADHD) are commonly associated with the FMR1 premutation.

- In premutation carriers with fragile-X-associated tremor/ataxia syndrome, anxiety is common and is associated with an increase in hippocampal atrophy.

- Psychosocial stresses could increase the risk of developing anxiety and depression in premutation carriers.

- ADHD and chronic pain symptoms can potentially lead to chronic substance abuse in premutation carriers.

\section{References}

Papers of special note have been highlighted as: $\bullet$ of interest; $\bullet \bullet$ of considerable interest

1. Tassone F, Iong KP, Tong TH et al. FMR1 CGG allele size and prevalence ascertained through newborn screening in the United States. Genome Med. 4(12), 100 (2012).

2. Tassone F, Hagerman RJ, Taylor AK, Gane LW, Godfrey TE, Hagerman PJ. Elevated levels of FMR1 mRNA in carrier males: a new mechanism of involvement in the fragile-X syndrome. Am. J. Hum. Genet. 66(1), 6-15 (2000).

3. Sherman SL. Premature ovarian failure in the fragile X syndrome. Am. J. Med. Genet. 97(3), 189-194 (2000).

4. Sullivan SD, Welt C, Sherman S. FMR1 and the continuum of primary ovarian insufficiency. Semin. Reprod. Med. 29(4), 299-307 (2011).

5. Jacquemont $\mathrm{S}$, Hagerman RJ, Leehey MA et al. Penetrance of the fragile X-associated tremor/ataxia syndrome in a premutation carrier population. JAMA 291(4), 460-469 (2004).

-. The first to describe the penetrance of tremor and ataxia in the premutation population of California.

6. Hagerman RJ, Leavitt BR, Farzin F et al. Fragile-X-associated tremor/ataxia syndrome (FXTAS) in females with the $F M R 1$ premutation. Am. J. Hum. Genet. 74(5), 1051-1056 (2004).

7. Rodriguez-Revenga L, Madrigal I, Pagonabarraga J et al. Penetrance of $F M R 1$ premutation associated pathologies in fragile X syndrome families. Eur. J. Hum. Genet. 17(10), 1359-1362 (2009).

8. Hagerman RJ, Protic D, Rajaratnam A, Salcedo-Arellano MJ, Aydin EY, Schneider A. Fragile X-associated neuropsychiatric disorders (FXAND). Front. Psychiatry (2018). https://www.ncbi.nlm.nih.gov/pmc/articles/PMC6243096/

- This is the first report to describe the several neuropsychiatric disorders that are associated with the premutation.

9. Tassone F, Pan R, Amiri K, Taylor AK, Hagerman PJ. A rapid polymerase chain reaction-based screening method for identification of all expanded alleles of the fragile X (FMRI) gene in newborn and high-risk populations. J. Mol. Diagn. 10(1), 43-49 (2008). 
10. Filipovic-Sadic S, Sah S, Chen L et al. A novel FMR1 PCR method for the routine detection of low abundance expanded alleles and full mutations in fragile X syndrome. Clin. Chem. 56(3), 399-408 (2010).

11. Famula JL, McKenzie F, McLennan YA et al. Presence of middle cerebellar peduncle sign in FMR1 premutation carriers without tremor and ataxia. Front. Neurol. 9, 695 (2008).

12. Hagerman RJ, Hagerman P. Fragile X-associated tremor/ataxia syndrome - features, mechanisms and management. Nat. Rev. Neurol. 12(7), 403-412 (2016).

13. Roberts JE, Bailey DB, Jr, Mankowski J et al. Mood and anxiety disorders in females with the FMR1 premutation. Am. J. Med. Genet. B Neuropsychiatr. Genet. 150B(1), 130-139 (2009).

- An important paper describing the prevalence of mood and anxiety disorders in women with the premutation.

14. Polussa J, Schneider A, Hagerman R. Molecular advances leading to treatment implications for fragile X premutation carriers. Brain Disord. Ther. 3:1000119 (2014).

15. Robin G, López JR, Espinal GM, Hulsizer S, Hagerman PJ, Pessah IN. Calcium dysregulation and Cdk5-ATM pathway involved in a mouse model of fragile X-associated tremor/ataxia syndrome. Hum. Mol. Genet. 26(14), 2649-2666 (2017).

16. Giulivi C, Napoli E, Tassone F, Halmai J, Hagerman R. Plasma metabolic profile delineates roles for neurodegeneration, pro-inflammatory damage and mitochondrial dysfunction in the FMR1 premutation. Biochem. J. 473(21), 3871-3888 (2016).

17. Napoli E, Ross-Inta C, Song G et al. Premutation in the fragile X mental retardation 1 (FMR1) gene affects maternal Zn-milk and perinatal brain bioenergetics and scaffolding. Front. Neurosci. 10, 159 (2016).

18. Sellier C, Buijsen RAM, He F et al. Translation of expanded CGG repeats into FMRpolyG is pathogenic and may contribute to fragile $\mathrm{X}$ tremor ataxia syndrome. Neuron 93(2), 331-347 (2017).

19. Brown SSG, Whalley HC, Kind PC, Stanfield AC. Decreased functional brain response to emotional arousal and increased psychiatric symptomology in FMR1 premutation carriers. Psychiatry Res. Neuroimaging 285, 9-17 (2019).

-• This interesting paper studies psychopathology in male carriers without fragile $X$-associated tremor/ataxia syndrome compared with controls. It also demonstrates that $\mathrm{fMRI}$ activation in carriers is significantly lower than in controls. They discuss this finding as a developmental disorder, which does not change in adult life from 24 to 70 years.

20. Bourgeois JA, Seritan AL, Casillas EM et al. Lifetime prevalence of mood and anxiety disorders in fragile X premutation carriers. J. Clin. Psychiatry 72(2), 175-182 (2011).

21. Franke $\mathrm{P}$, Leboyer $\mathrm{M}$, Gansicke $\mathrm{M}$ et al. Genotype-phenotype relationship in female carriers of the premutation and full mutation of FMR-1. Psychiatry Res. 80(2), 113-127 (1998).

22. Roberts JE, Tonnsen BL, McCary LM, Ford AL, Golden RN, Bailey DB, Jr. Trajectory and predictors of depression and anxiety disorders in mothers with the FMR1 premutation. Biol. Psychiatry 79(10), 850-857 (2016).

-. This excellent paper studies mothers with the premutation and demonstrates that the prevalence of depression and anxiety disorders increased with age in their cohort of women carriers.

23. Cordeiro L, Abucayan F, Hagerman R, Tassone F, Hessl D. Anxiety disorders in fragile X premutation carriers: preliminary characterization of probands and non-probands. Intractable Rare Dis. Res. 4(3), 123-130 (2015).

24. Sobesky WE, Pennington BF, Porter D, Hull CE, Hagerman RJ. Emotional and neurocognitive deficits in fragile X. Am. J. Med. Genet. 51(4), 378-385 (1994).

25. Farzin F, Perry H, Hessl D et al. Autism spectrum disorders and attention-deficit/hyperactivity disorder in boys with the fragile $\mathrm{X}$ premutation. J. Dev. Behav. Pediatr. 27(2 Suppl), S137-S144 (2006).

- This is one of the first to document the high incidence of psychopathology in boys with the premutation who were probands versus their nonproband carrier brothers versus brothers who are not carriers.

26. Bailey DB, Jr, Raspa M, Olmsted M, Holiday DB. Co-occurring conditions associated with FMR1 gene variations: findings from a national parent survey. Am. J. Med. Genet. 146A(16), 2060-2069 (2008).

- This documents a large survey involving psychiatric diagnoses in those with fragile $X$ syndrome and those who are carriers, and it documents the high prevalence of problems in boys with the premutation compared with girls with the premutation.

27. Wadell PM, Hagerman RJ, Hessl DR, Fragile X. Syndrome: psychiatric manifestations, assessment and emerging therapies. Curr. Psychiatry Rev. 9(1), 53-58 (2013).

28. Hunter JE, Epstein MP, Tinker SW, Abramowitz A, Sherman SL. The FMR1 premutation and attention-deficit hyperactivity disorder (ADHD): evidence for a complex inheritance. Behav. Genet. 42(3), 415-422 (2012).

29. Coffey SM, Cook K, Tartaglia N et al. Expanded clinical phenotype of women with the FMR1 premutation. Am. J. Med. Genet. A 146A(8), 1009-1016 (2008).

30. Leehey MA, Legg W, Tassone F, Hagerman R. Fibromyalgia in fragile X mental retardation 1 gene premutation carriers. Rheumatology 50(12), 2233-2236 (2011).

31. Dorn MB, Mazzocco MMM, Hagerman RJ. Behavioral and psychiatric disorders in adult male carriers of fragile X. J. Am. Acad. Child Adolesc. Psychiatry 33(2), 256-264 (1994). 
32. Kogan CS, Turk J, Hagerman RJ, Cornish KM. Impact of the fragile X mental retardation 1 (FMR1) gene premutation on neuropsychiatric functioning in adult males without fragile X-associated tremor/ataxia syndrome: a controlled study. Am. J. Med. Genet. B Neuropsychiatr. Genet. 147B(6), 859-872 (2008).

33. Besterman AD, Wilke SA, Mulligan TE et al. Towards an understanding of neuropsychiatric manifestations in fragile $\mathrm{X}$ premutation carriers. Future Neurol. 9(2), 227-239 (2014).

34. Wheeler AC, Bailey DB, Jr, Berry-Kravis E et al. Associated features in females with an FMR1 premutation. J. Neurodev. Disord. 6(1), 30 (2014).

35. Song G, Napoli E, Wong $S$ et al. Altered redox mitochondrial biology in the neurodegenerative disorder fragile X-tremor/ataxia syndrome: use of antioxidants in precision medicine. Mol. Med. 22, 548-559 (2016).

36. Wang JY, Hessl D, Hagerman RJ et al. Abnormal trajectories in cerebellum and brainstem volumes in carriers of the fragile $\mathrm{X}$ premutation. Neurobiol. Aging 55, 11-19 (2017).

- An excellent cross-sectional study of brain volume changes of carriers over the lifespan demonstrating that these problems begin in childhood compared with controls.

37. Conde V, Palomar FJ, Lama MJ et al. Abnormal GABA-mediated and cerebellar inhibition in women with the fragile X premutation. J. Neurophysiol. 109(5), 1315-1322 (2013).

- An important paper documenting GABA deficits in premutation carriers that impact inhibitory processes.

38. Hamlin A, Liu Y, Nguyen DV, Tassone F, Zhang L, Hagerman RJ. Sleep apnea in fragile X premutation carriers with and without FXTAS. Am. J. Med. Genet. B Neuropsychiatr. Genet. 156B(8), 923-928 (2011).

39. Bourgeois JA, Coffey SM, Rivera SM et al. A review of fragile X premutation disorders: expanding the psychiatric perspective. J. Clin. Psychiatry 70(6), 852-862 (2009). 